\title{
Using Cluster Analysis to Explore Associations between Cardiovascular Risk and Lifestyle Factors in a Workplace Wellness Program
}

\author{
Heather Carter-Templeton, PhD, RN-BC, FAAN \\ West Virginia University \\ Gary Templeton, PhD \\ West Virginia University \\ Barbara Ann Graves, PhD, RN \\ The University of Alabama \\ Leslie G. Cole, DNP, RN \\ The University of Alabama
}

\begin{abstract}
Background: Cardiovascular disease (CVD) is the number one cause of death in the United States with risk factors including hypertension, hyperlipidemia, diabetes, obesity, smoking, physical inactivity, age, genetics, and unhealthy diets. A university-based workplace wellness program (WWP) consisting of an annual biometric screening assessment with targeted, individualized health coaching was implemented in an effort to reduce these risk factors while encouraging and nurturing ideal cardiovascular health.

Objective: The purpose of this study was to examine and describe the prevalence of single and combined, or multiple, CVD risk factors within a workplace wellness dataset.

Methods: Cluster analysis was used to determine CVD risk factors within biometric screening data (BMI, waist circumference, LDL, total cholesterol, HDL, triglycerides, blood glucose age, ethnicity, and gender) collected during WWP interventions.

Results: The cluster analysis provided visualizations of the distributions of participants having specific CVD risk factors. Of the 8,802 participants, 1,967 (22.4\%) had no CVD risk factor, 1,497 (17\%) had a single risk factor, and 5,529 (60.5\%) had two or more risk factors. The majority of sample members are described as having more than one CVD risk factor with $78 \%$ having multiple.

Conclusion: Cluster analysis demonstrated utility and efficacy in categorizing participant data based on their CVD risk factors. A baseline analysis of data was captured and provided understanding and awareness into employee health and CVD risk. This process and analysis facilitated WWP planning to target and focus on education to promote ideal cardiovascular health.
\end{abstract}

Submitted 25 September 2020: accepted 25 November 2020

Keywords: cardiovascular risk factors, cluster analysis, health promotion

Cardiovascular disease (CVD) is the leading cause of death and chronic illness in the United States (US). The burden of CVD is substantial in terms of mortality, disability, and cost. More than 92 million Americans (1 in 3) have some 
type of CVD (Benjamin et al., 2017). The age-standardized death rate for all CVDs in the US population was 220.8 per 100,000 in the year 2014. In 2012 to 2013, the total direct and indirect costs of CVD and stroke was an estimated $\$ 316.1$ billion. It is projected that by the year 2030 more than $43 \%$ of the US adult population will have some type of CVD (Benjamin et al., 2017).

CVD is the number one cause of death in the US (Centers for Disease Control [CDC], 2016a; Go et al., 2014). Risk factors for CVD include hypertension, hyperlipidemia, diabetes, obesity, smoking, physical inactivity, age, genetics, and unhealthy diets (National Institutes of Health, 2018). Understanding the epidemiological risk factors that bring about disease is imperative to improving cardiovascular health (Arnett et al., 2019).

Research studies have revealed that CVD risk factors tend to cluster in some individuals. Rigorous clinical trials have discovered that individuals with clustered CVD risk factors are more prone to develop CVD when compared to those with a single CVD risk factor (Arnett et al., 2019). Furthermore, sedentariness and unhealthy diets, have been associated with increased risk of some chief modifiable lifestyle-related risk factors, and are conducive to the grouping of CVD risk factors (Arnett et al., 2019). Coexisting CVD risk factors exponentially increases the risk of developing CVD and complications.

One American Heart Association (AHA) Impact Goal for 2020 was "to improve the cardiovascular (CV) health of all Americans by 20\%, while reducing deaths from CVDs and stroke by 20\%”(Go et al., 2014, p. 12). High rates of CVD mortality are driven by the prevalence of CVD risk factors. Moreover, it is well known that risk factors tend to cluster in some individuals increasing their risk of CVD (Younus et al., 2016). A plethora of seminal research has demonstrated the effectiveness of CVD risk assessment and risk reduction through the years (Dawber Kannel, \& Lyell, 1963; Fried et al., 1991; Kannel et al., 1979). More recent population-based studies have been equally influential through examination of the prevalence and trends in CVD risk (Benjamin et al., 2017; Shay, Gooding, Murillo, \& Foraker, 2015).

Studies have documented worksite wellness programs (WWP) as an important strategy in overall health prevention and promotion (Carnethon et al., 2009; Milani \& Lavie, 2009; Osilla et al., 2102; Webber et al., 2012). WWPs are positioned to provide population-based strategies toward meeting AHA Impact Goals. They can focus on the health promotion of healthy behaviors and healthy metrics, in addition to population-level health promotion strategies aimed toward improving cardiovascular health and targeting individuals at the greatest CVD risk (Benjamin et al., 2017).

WellBAMA, the signature WWP at the University of Alabama (UA), is focused on advancing the health and wellbeing of its employees. At the heart of its mission is the reduction of health risk, prevention of disease, and promotion of ideal health. The program operates in a collaborative model utilizing partnerships with the Office of Health Promotion and Wellness, the Capstone College of Nursing, the College of Community Health Science, and the Division of Financial Affairs. The program provides onsite health screenings and assessments, individual health coaching sessions, and opportunities to participate in health-related programs. WellBAMA is available to all employees at the UA (Carter, Kelly, \& Alexander, 2011).

A WWP was introduced at a university in the Southeastern US in 2007. Initially, the program consisted of an annual biometric screening assessment with targeted, individualized health coaching. Biometric data collected during annual wellness screenings included measurable health data: body mass index (BMI), blood pressure (BP), low density lipoprotein (LDL), total cholesterol, high density lipoprotein (HDL), triglycerides, and glucose. Participants were assigned a "risk category" based on their wellness score. Risk categories were assigned financial incentive amounts. 
Additional educational components to increase knowledge and engage participants were added to annual wellness screenings. Prior to these additions, participants were financially rewarded for simply participating in the annual health screening with individualized health coaching. With the addition of required educational group sessions and qualifying programs, financial incentives then became contingent on participation in both screening and educational sessions. These added components have resulted in positive trends among individual biometric variables. Program effectiveness is currently determined through aggregate biometric data tracking and longitudinal analysis. Though this evaluation does show positive trends in biometric variables over time, analyses have been unable to determine the nature, relationship, and prevalence of individual biometric measurements when clustered with coexisting variables.

Independently examining biometric data trends over time gives a limited, one-dimensional perspective to biometric health data that is often influenced by other health variables. Considering the health impact of comorbid risk factors in CVD, clustering data could provide valuable information about employee risk while giving insight into additional wellness initiatives that may accurately target health needs of high-risk participants.

The purpose of this retrospective, descriptive, secondary analysis was to examine the prevalence of single, concurrent, and clustered CVD risk factors in a worksite wellness program using a cluster analysis. This study sought to describe and add an understanding of promoting cardiovascular health in the context of this specific WWP through the assessment of cardiovascular metrics. Cluster analysis can assist in discovering the classifications and relationships within and among homogeneous groups (Dunn et al., 2017) to further investigate CVD risk. The aim was to identify the most prevalent and clustered risk factors or metrics to guide strategy and program development to target CVD prevention and promote cardiovascular health.

\section{METHODS}

Investigators obtained approval from the university Institutional Review Board to analyze existing WWP biometric data from employees who underwent health screenings from January 2011 to December 2018. De-identified data for the defined study period was securely exported from the university institutional technology department to a passwordprotected Microsoft Excel file for use in analyses. The data were originally collected by nursing students under supervision of nursing faculty and the Health and Wellness Office Clinician and Researcher for the university as a part of the community health clinical experiences for the students. Inclusion criteria were: (1) full-time university employees eligible for benefits; (2) greater than or equal to 18 years of age; and (3) completion of the screening within the stated eight-year timeframe. All findings were safely stored and saved by the university institutional technology department.

Three types of data were used in the study. Demographic variables included age, gender, and ethnicity. Also, five anthropometric data representing CVD risk factors were assessed: (1) hypertension (systolic and diastolic blood pressure); (2) diabetes (glucose levels); (3) hyperlipidemia (LDL cholesterol and triglycerides); 4) overweightness (body mass index); and (5) abdominal obesity (waist circumference). Measures used to assess lifestyle habits included extent of cardio exercise, extent of tobacco use, and need for physical or eye examination. Among CVD risk measures, low density lipoprotein (LDL) was selected over high-density lipoprotein (HDL) as it can be a more decisive tool in diagnosing hypercholesterolemia in the clinical setting (AHA, 2018). Elevated total cholesterol was not used because the increase in this level could be in response to increased HDL blood levels.

Subjects were classified as having a specific CVD risk by meeting accepted thresholds within a given risk factor. Four clusters of subjects were defined, i.e., those having 0, 1, 2 or 3 or more CVD risk. As reported in Table 1, the 
thresholds for specific criteria were designated per the published guidelines from national organizations. CVD criteria thresholds were also used by the university WWP when assigning a wellness risk category status for participants.

Table 1. Study CVD Risk Factor Thresholds and Sources

\begin{tabular}{|l|l|l|}
\hline CVD Risk Factor & Threshold(s) & Source \\
\hline Hypertension & $\begin{array}{l}\text { Systolic BP } \geq 130 \text { or } \\
\text { Diastolic BP } \geq 80\end{array}$ & AHA, 2017 \\
\hline Diabetes & Fasting Glucose $\geq 100$ & ADA, 2016 \\
\hline Hyperlipidemia & $\begin{array}{l}\text { LDL Cholesterol } \geq 140 \text { or } \\
\text { Triglyceride } \geq 150\end{array}$ & NIH, 2018 \\
\hline $\begin{array}{l}\text { Overweight (Waist } \\
\text { Circumference in inches) }\end{array}$ & Body Mass Index (BMI) $\geq 25$ & CDC, 2016a \\
\hline
\end{tabular}

Note. AHA = American Heart Association; ADA = American Diabetes Association; NIH = National Institute of Health; $\mathrm{CDC}=$ Centers for Disease Control

Cluster analysis is a descriptive (Lindgren et al., 2008) approach to data analysis used in support of visualization, pattern recognition, and artificial intelligence. It is used to identify and label groups (i.e., clusters) of subjects displaying similar characteristics or tendencies based on a designated set of theoretically important variables. Thus, cluster analysis is any approach using observational data to classify records. This differs from discriminant analysis, where groups are already known as a consequence of data collection (Kaufman \& Rouseeuw, 1990). Beckstead (2002) enumerates a series of steps normally performed during cluster analysis: (1) selecting the sample; (2) defining variables; (3) calculating the measure of similarity; (4) grouping units together based on their measures of similarity using an algorithm; (5) creating an illustration or graphic to assist in interpretation of the results; (6) and validating the results. Understanding and interpreting resulting clusters profoundly depends on the researchers' understanding of the data and study context (Perrin, 2004). Rather than using automated software for this purpose, we followed Gao, Zhang, and Wang (2013) in establishing clusters using criteria thresholds.

A Microsoft Excel-based dashboard was developed to store, evaluate, and expedite quality control over the study data. Subject matter experts on the project team worked collaboratively to iteratively clean the data and evaluate the logic of cluster assignment based on CVD risk criteria and according to practice guidelines (Arnett et al., 2019). Based on four cluster groupings, descriptive statistics were calculated.

\section{RESULTS}

Shown in Table 2, the cluster analysis illuminated the distribution of participants according to demographics and across levels of CVD risk. After the participation constraints were applied, the final sample consisted of 8,802 biometric data records. In this dataset, both genders were represented with participants being predominantly female (70\%). A variety of ethnicities were also represented: less than 1\% American Indian/Alaskan Native $(n=16) ; 4 \%$ Asian/Pacific Islander $(n=352) ; 17 \%$ Black/African American $(n=1,469)$; less than 1\% Hispanic $(n=113)$; less than $1 \%$ undeclared/other $(n=2)$; and $78 \%$ White $(n=6749)$. Lastly, the descriptive statistics indicated that participant ages (in years) ranged from 18 to 82 with the average age being 41.7 . 
Table 2 also details the distribution of participants across the cluster assignments. Of the study participants, 1,976 (22.4\%) had no CVD risk factor, 1,497 (17.0\%) had a single risk factor, 1,718 (19.5\%) had two risk factors, and 3,611 $(41.0 \%)$ had three or more risk factors. This result can also be interpreted as 5,329 (60.5\%) of participants having more than one CVD risk factor. Further, among the 6,826 participants having at least one risk factor, 78.0\% had multiple. The Venn diagram (Figure 1) depicts the proportional relationships among sample members based on occurrences of the five selected CVD risk factors. The gray area is the part of the sample with no CVD risk factors. The non-gray/unblended colors represent regions of a single risk factor while non-gray/blended colors represent participants having multiple attributes. The numbers represent the subject count having CVD risk factors defined for the primary color-specific category. Figure 1 is useful for showing the distribution of types of participants pertaining to having or not having specific risk factors. This figure illustrates that a majority of sample members are described as having more than one CVD risk factor.

Our data also indicated the rate at which sample members met specific CVD risk thresholds. Participants being high in systolic BP $(n=1,809 ; 20.6 \%)$ and diastolic BP $(n=3476 ; 39.5 \%)$ resulted in $n=3,741(42.5 \%)$ meeting the criteria for hypertension. Diabetes was an issue in 1,860 (21.2\%) of participants scoring glucose levels higher than the threshold value. Participants meeting or exceeding the thresholds for LDL cholesterol $(n=1,017 ; 11.6 \%)$ and triglycerides $(n=1,969 ; 22.4 \%)$ resulted in $n=2,616$ (29.8\%) exhibiting hyperlipidemia. Additionally, it was evident that the majority $(n=5783 ; 65.7 \%$ ) of participants were at risk by being overweight (as defined by a Body Mass Index $>25)$. Evidence shows that $37.0 \%(n=977)$ of males and $46.7 \%(n=2,875)$ of females met or surpassed the threshold for abdominal obesity (waist circumference).

Table 2 also alludes to relationships between CVD risk (as defined by cluster membership level) and other characteristics of participants. For example, males and older participants had an increased prevalence of clustered CVD risks. There were also health disparities in terms of ethnicity as CVD risk factors increased. For example, participants classified as American Indian/Native Alaskan or Black/African American had greater prevalence rates as the number of CVD risks increased, while those classified as Asian/Pacific Islander and White had lower prevalence rates as the number of CVD risks increased. Further, Table 2 shows that greater levels of cardiovascular exercise reduce the prevalence of CVD risk. Within each cluster group $(0,1,2, \geq 3)$, the cardio distribution tended toward greater cardio levels in the sample. However, this distribution became more uniform for subjects in higher risk clusters. Only risk clusters $0(79.1 \%)$ and $1(58.8 \%)$ contained a majority of participants reporting a Cardio Level of 4 . Therefore, this sample provided strong evidence that higher cardio physical activity levels relate to lower CVD risk. Additionally, Table 2 displays that tobacco use consistently influences CVD risk cluster membership.

For example, as risk clusters increased, the rate at which participants reported that they "Never Used or Quit for 12 months" decreased (i.e., $0=97.7 \%$ and $\geq 3=90.9 \%$ ). By contrast, as risk clusters increased, the rate at which participants reported "Current or Quitting tobacco $<6$ Months" also increased (i.e., $0=1.9 \%$; $\geq 3=8.0 \%$ ). Finally, those reporting "Needs a Physical Exam" and "Needs an Eye Exam" both presented as having uniform distributions across cluster membership.

Age (in years) was operationalized for both a balanced subject size among the groups and interval consistency. Age groups were labeled based on exact range midpoints, with exception to the last group: 17.5 (15-20); 22.5 (20-25); 27.5 (25-30); 32.5 (30-35); 37.5 (35-40); 42.5 (40-45); 47.5 (45-50); 52.5 (50-55); 57.5 (55-60); 62.5 (60-65); and 67.5 (65-82). Figure 2 shows the rate of risk cluster prevalence among female and male participants across the distribution 
of age. Both genders exhibit an increase in risk cluster prevalence as age advances and the prevalence of males is consistently greater than that of females across all age groups.

Table 2: Characteristics of Participants According to CVD Risk Factors

\begin{tabular}{|c|c|c|c|c|c|}
\hline & \multicolumn{4}{|c|}{ Cluster Membership } & \multirow{2}{*}{ Total } \\
\hline & 0 & 1 & 2 & $\geq 3$ & \\
\hline Number & 1976 & 1497 & 1718 & 3611 & 8802 \\
\hline Prevalence $(\%)$ & $22.4 \%$ & $17.0 \%$ & $19.5 \%$ & $41.0 \%$ & \\
\hline \multicolumn{6}{|l|}{ Age (years) } \\
\hline 17.5 & $0.4 \%$ & $0.3 \%$ & $0.6 \%$ & $0.2 \%$ & 32 \\
\hline 22.5 & $7.3 \%$ & $7.8 \%$ & $6.3 \%$ & $4.0 \%$ & 515 \\
\hline 27.5 & $17.5 \%$ & $16.8 \%$ & $13.4 \%$ & $10.4 \%$ & 1,200 \\
\hline 32.5 & $17.6 \%$ & $17.2 \%$ & $16.9 \%$ & $12.9 \%$ & 1,361 \\
\hline 37.5 & $17.3 \%$ & $15.1 \%$ & $13.0 \%$ & $13.5 \%$ & 1,277 \\
\hline 42.5 & $11.2 \%$ & $11.3 \%$ & $11.4 \%$ & $14.2 \%$ & 1,099 \\
\hline 47.5 & $7.1 \%$ & $8.9 \%$ & $11.8 \%$ & $14.7 \%$ & 1,007 \\
\hline 52.5 & $10.2 \%$ & $10.1 \%$ & $10.9 \%$ & $13.5 \%$ & 1,026 \\
\hline 57.5 & $7.0 \%$ & $7.7 \%$ & $9.1 \%$ & $10.2 \%$ & 776 \\
\hline 62.5 & $3.5 \%$ & $3.5 \%$ & $5.0 \%$ & $4.8 \%$ & 381 \\
\hline 67.5 & $0.9 \%$ & $1.2 \%$ & $1.5 \%$ & $1.6 \%$ & 119 \\
\hline Male & $18.9 \%$ & $30.7 \%$ & $33.7 \%$ & $34.1 \%$ & 2,644 \\
\hline \multicolumn{6}{|l|}{ Ethnicity } \\
\hline American Indian/Alaska Native & $0.1 \%$ & $0.0 \%$ & $0.2 \%$ & $0.3 \%$ & 16 \\
\hline Asian or Pacific Islander & $6.1 \%$ & $5.5 \%$ & $4.3 \%$ & $2.1 \%$ & 352 \\
\hline Black or African-American & $5.6 \%$ & $10.9 \%$ & $18.2 \%$ & $24.5 \%$ & 1,469 \\
\hline Hispanic & $1.5 \%$ & $0.9 \%$ & $1.5 \%$ & $1.2 \%$ & 113 \\
\hline Undeclared/other & $0.0 \%$ & $0.1 \%$ & $0.0 \%$ & $0.0 \%$ & 2 \\
\hline White & $85.5 \%$ & $80.8 \%$ & $74.2 \%$ & $71.1 \%$ & 6,743 \\
\hline \multicolumn{6}{|l|}{ Exercise } \\
\hline Cardio Level 1 & $2.9 \%$ & $5.6 \%$ & $8.7 \%$ & $14.4 \%$ & 811 \\
\hline Cardio Level 2 & $6.3 \%$ & $10.3 \%$ & $15.4 \%$ & $19.5 \%$ & 1,247 \\
\hline Cardio Level 3 & $11.6 \%$ & $25.3 \%$ & $26.3 \%$ & $31.6 \%$ & 2,200 \\
\hline Cardio Level 4 & $79.1 \%$ & $58.8 \%$ & $49.7 \%$ & $34.5 \%$ & 4,544 \\
\hline \multicolumn{6}{|l|}{ Tobacco Use } \\
\hline Current or Quit $<6$ Months & $1.9 \%$ & $3.7 \%$ & $4.8 \%$ & $8.0 \%$ & 462 \\
\hline Quit for between 6 and 12 Months & $0.4 \%$ & $0.5 \%$ & $0.9 \%$ & $1.1 \%$ & 71 \\
\hline Never Used or Quit for 12 Months & $97.7 \%$ & $95.9 \%$ & $94.3 \%$ & $90.9 \%$ & 8,263 \\
\hline Needs Physical Exam & $18.9 \%$ & $18.4 \%$ & $18.9 \%$ & $17.8 \%$ & 1,617 \\
\hline Needs Eye Exam & $19.0 \%$ & $20.0 \%$ & $18.7 \%$ & $20.2 \%$ & 1,653 \\
\hline
\end{tabular}


Figure 1. Distribution of Five Major CVD Risk Factors in Sample

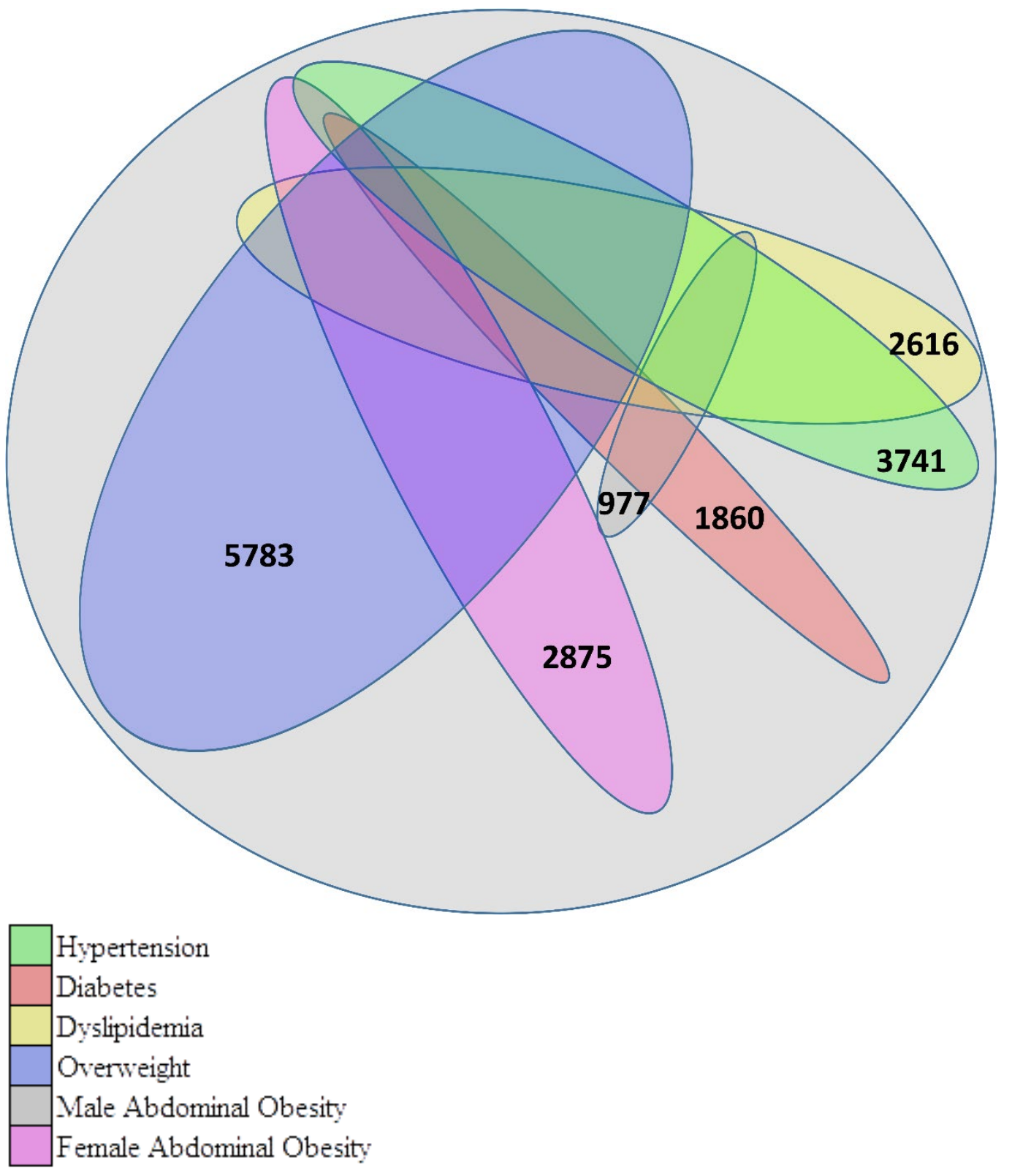


Figure 2. Rate of Risk Cluster Prevalence by Gender across Age Groups

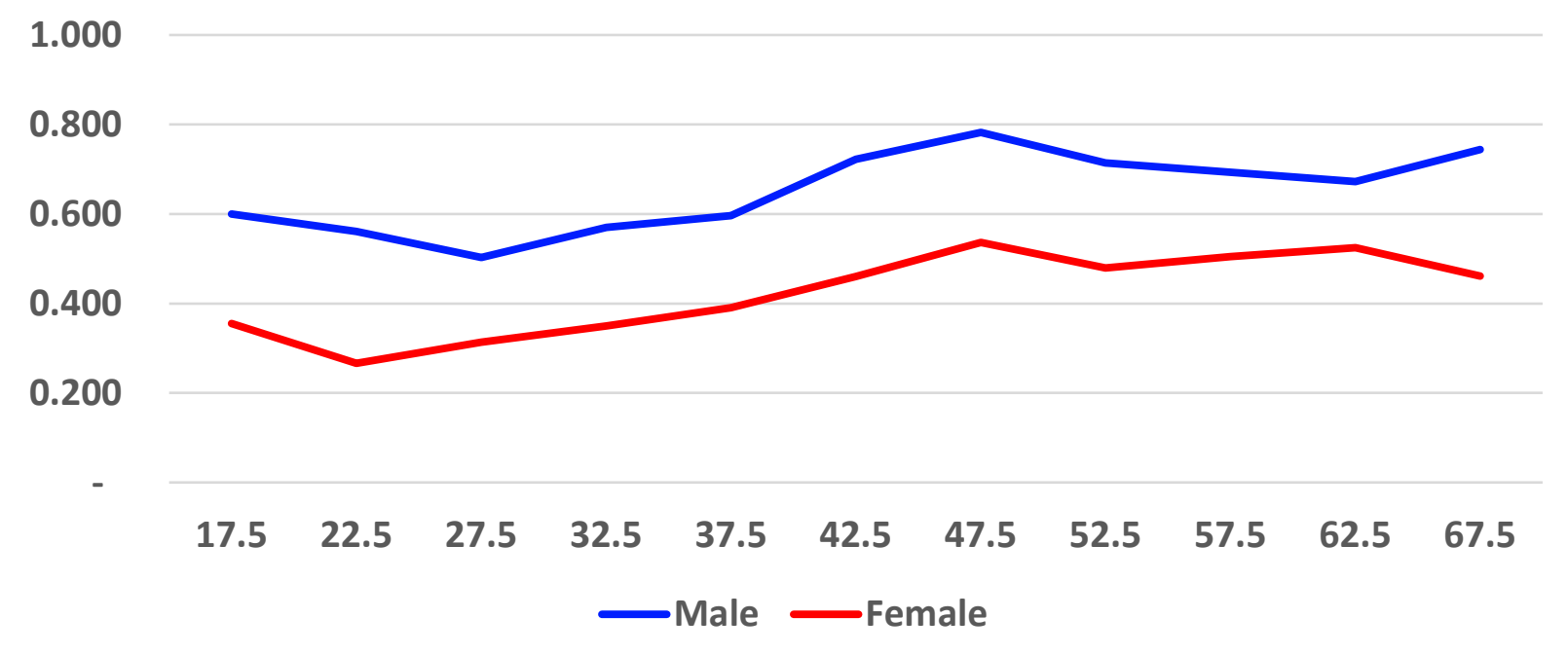

Figure 3 shows the rate of risk cluster prevalence by ethnicity across age groups. Black/African American participants consistently exhibited the highest risk cluster prevalence alone and by age increase. The data also denotes that White participants have rate increases of risk cluster prevalence by age increase. Asian/Pacific Islander participants were generally lower in risk cluster prevalence than the other ethnicities, except in later years.

Figure 3. Rate of Risk Cluster Prevalence by Race across Age Groups

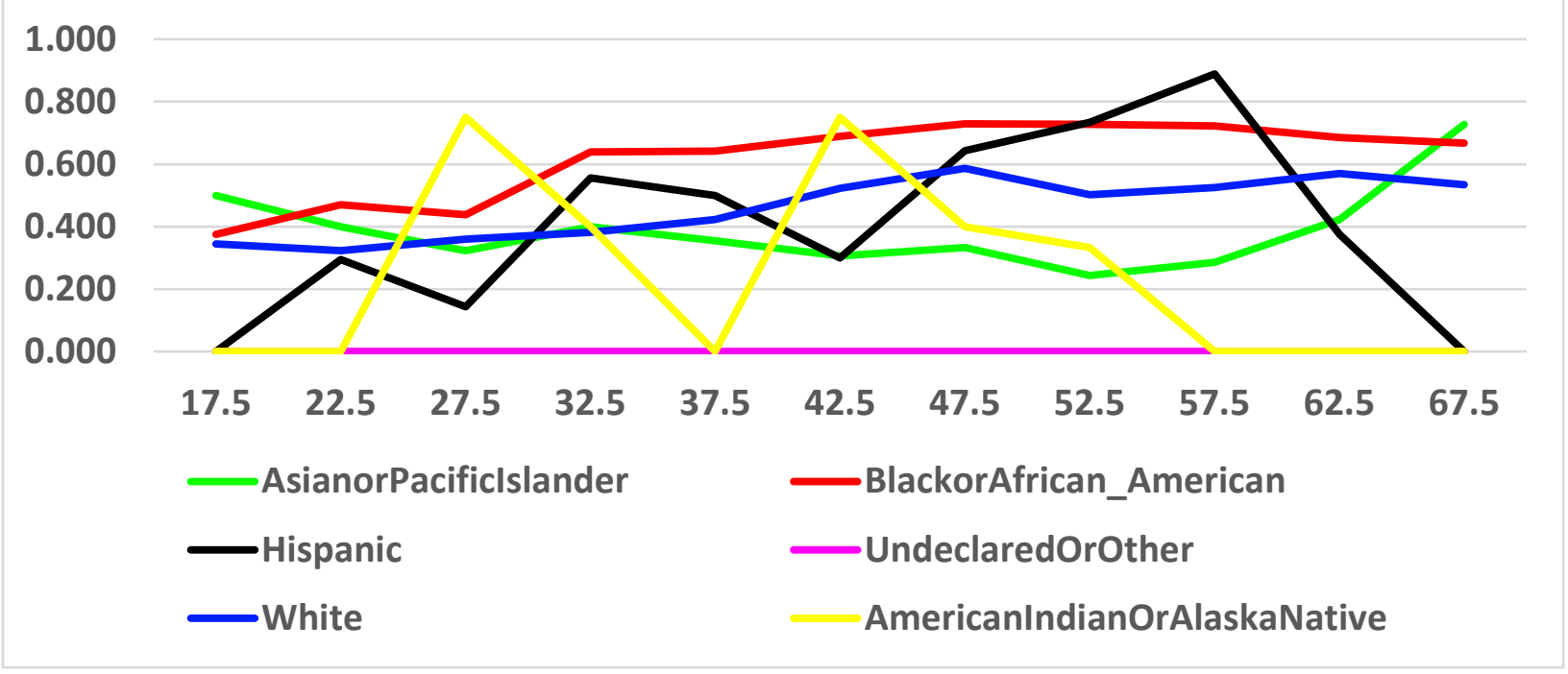

\section{DISCUSSION}

The clustering of the following five major CVD risk factors were observed: (1) hypertension; (2) diabetes; (3) hyperlipidemia; (4) overweight; and abdominal obesity. In this university WWP sample, clustering was prominent among major modifiable lifestyle-related risk factors known to highly influence the development of CVD. Results 
indicated that over half of the participants with existing CVD risk factors had clustering risk factors. Of the participants having one or more risk factors, more than three-fourths had clustering risk factors.

Men in this study had a higher rate of CVD risk clustering prevalence than women. This finding has been demonstrated historically in the prevalence of many single CVD risk factors (Arnett et al., 2019). Most notable this study revealed a high prevalence of participants with Body Mass Index > 25 (overweight) and abdominal obesity, both of which are considered CVD risk factors (Arnett et al., 2019).

Identification of CVD risk factor clustering is valuable for determining overall risk and designing targeted prevention and intervention strategies. Many research studies have shown that comprehensive lifestyle interventions are effective strategies for controlling CVD risk. Furthermore, research has documented that adhering to a healthy lifestyle is associated with lowering CVD risk (Arnett et al., 2019). Thus, it is important for WWP to encouraging healthy lifestyles to both prevent and decrease the prevalence of CVD risk factors. WWPs are ideal arenas for the development of strategies and incentives in the areas of diet and physical activity to help modify these lifestyle-related risks.

In is important to note that one research team member was a health promotion expert working within the WWP. This added valuable insight into the context in which the data was collected and used. Perrin (2004) suggested that this understanding of data and context is essential to the interpretation and aided in ensuring the quality and credibility of the analysis.

\section{CONCLUSION}

Cluster analysis proved useful in grouping participant data based on their CVD risk factors. A baseline analysis of data was conducted and an understanding of CVD risk clustering in this specific population was gained. This analysis provided insight into employee health and CVD risk, allowing those that plan educational activities and interventions to specifically target and address prevalent CVD risk factors and combinations thereof among the participants in the WWP. The development of customized specific risk reduction strategies, interventions, and programs could improve poor health consequences often associated with CVD risk factors.

Historically, cluster analysis has proven to be a valuable means for clinicians to identify and address symptom management. Likewise, this analysis was helpful in identifying employee CVD risk factors and clustering of risk. This analysis offered a basic classification scheme derived from the data and improves the ability for the WWP to categorize an employee into a specific cluster with customized interventions and educational information designed to reduce CVD risk. Furthermore, the easily accessible dashboard created for this research allows for future monitoring of effectiveness of interventions among and within each cluster.

Moving forward, it is important to further develop risk assessments into a positive framework of ideal cardiovascular health (iCVH) metrics (Benjamin et al., 2017). New and more current guidelines and strategies (Benjamin et al., 2017; Shay, Gooding, Murillo, \& Foraker, 2015; Yank et al., 2012; Younus et al., 2016) will need to be adapted to expand the focus on CVD prevention from simple risk reduction to the promotion of positive "cardiovascular health" as characterized by seven health metrics. Within this focus, the new iCVH can be measured not only the absence of clinical CVD but also with the presence of "optimal levels" of four healthy behaviors (not smoking, having sufficient physical activity, a healthy diet pattern, and appropriate energy balance or normal body weight) and three health factors (optimal total cholesterol, blood pressure, and fasting glucose, in the absence of drug treatment (Benjamin et al., 2017). 


\section{REFERENCES}

American Diabetes Association (ADA). (2016). Diagnosing Diabetes and Learning about Prediabetes. Retrieved from https://medlineplus.gov/triglycerides.html

American Heart Association (AHA). (2017). Understanding Blood Pressure Readings. Retrieved from https://www.heart.org/en/health-topics/high-blood-pressure/understanding-blood-pressure-readings

American Heart Association (AHA). (2018). About Metabolic Syndrome. Retrieved from http://www.heart.org/en/health-topics/metabolic-syndrome/about-metabolic-syndrome

Arnett D.K., Blumenthal R.S., Albert M.A., Buroker, A.B., Goldberger, Z.d., Hahn, E. J., ... Ziaeian, B. (2019). 2019 ACC/AHA Guideline on the Primary Prevention of Cardiovascular Disease: A Report of the American College of Cardiology/American Heart Association Task Force on Clinical Practice Guidelines. Journal of the American College of Cardiology, 74(10), e177-e232. https://doi.org/10.1161/CIR.0000000000000678

Beckstead, J.W. (2002). Using Hierarchical Cluster Analysis in Nursing Research. Western Journal of Nursing Research. 24(3):307-319. https:/ / doi.org/10.1177/01939450222045923

Benjamin, E.J., Blaha, M.J., Chiuve, S.E., Cushman, M., Das Sandeep, R. Deo, R., ... Munter, P. (2017). Heart disease and stroke statistics_-2017 update: A report from the American Heart Association. Circulation, 135(10): e146-e603. https://doi.org/10.1161/CIR.0000000000000485.

Carter, M.R., Kelly, R. \& Alexander, C. WellBama: A collaborative university model for employee health and wellness. American Journal of College Health. 2011;59:761-763. https://doi.org/10.1080/07448481.2010.544347

Carnethon. M, Whitsel, L. P., Franklin, B. A., Kris-Etherton, P., Milani, R., Pratt, C. A., \& Wagner, G.R. (2009). Worksite wellness programs for cardiovascular disease prevention: A policy statement from the American Heart Association. Circulation. 120(17):1725-1741. https://doi.org/10.1161/CIRCULATIONAHA.109.192653

Centers for Disease Control (CDC). (2016a). Defining Adult Overweight and Obesity. Retrieved from https://www.cdc.gov/obesity/adult/defining.html

Centers for Disease Control (CDC). (2016b). Leading Causes of Death. Retrieved from: https://www.cdc.gov/nchs/fastats/leading-causes-of-death.htm

Dawber, T.R., Kannel, W.B, Lyell, L.P. (1963). An approach to longitudinal studies in a community: the Framingham Study. Ann N Y Acad Sci.107:539-556. https://doi.org/10.1111/j.1749-6632.1963.tb13299.x

Dunn, H., Quinn, L., Corbridge, S. J., Eldeirawi, K., Kapella, M., \& Collins, E. G. (2017). Cluster analysis in nursing research: An introduction, historical perspective, and future directions. Western Journal of Nursing Research, 40 (11), 1658-1676. https://doi.org/10.1177/0193945917707705

Fried, L.P., Borhani, N.O., Enright, P., et al. (1991). The Cardiovascular Health Study: design and rationale. Ann Epidemiol. 1:263-276. https://doi.org/10.1016/1047-2797(91)90005-W 
Gao, B., Zhang, L., Wang, H., \& China National Survey of Chronic Kidney Disease Working Group. (2013). Clustering of Major Cardiovascular Risk Factors and the Association with Unhealthy Lifestyles in the Chinese Adult Population. PLoS One 8(6): e66780. https://doi.org/10.1371/journal.pone.0066780

Go, A. S., Mozaffarian, D., Roger, V. L., Benjamin, E. J., Berry, J. D., Blaha, M. J., ...Turner, M. (2013). Heart disease and stroke statistics--2014 update: A report from the American Heart Association. Circulation, 129(3) https://doi.org/10.1161/01.cir.0000441139.02102.80

Kannel, W.B., Feinleib, M., McNamara, P.M, et al. (1979). An investigation of coronary heart disease in families. Am J Epidemiol. 110:281-290. https://doi.org/10.1093/oxfordjournals.aje.a112813

Kaufman, L., \& Rousseeuw, P.J. (1990) Finding groups in data: An introduction to cluster analysis. New York, John Wiley \& Sons. https://doi.org/10.1002/9780470316801

Lindgren, T. G., Fukuoka, Y., Rankin, S. H., Cooper, B. A., Carroll, D., \& Munn, Y. L. (2008). Cluster analysis of elderly cardiac patients' prehospital symptomology. Nursing Research 57(1): 14-22. https://doi.org/10.1097/01.NNR.0000280654.50642.1a

Milani, R.V., \& Lavie, C. J. (2009). Impact of worksite wellness intervention on cardiac risk factors and one-year health care costs. The American Journal of Cardiology. 104(10):1389-1392. https://doi.org/10.1016/j.amjcard.2009.07.007

National Institutes of Health. Lower Heart Disease Risk. (2018). https://www.nhlbi.nih.gov/health/educational/hearttruth/lower-risk/risk-factors.htm. Accessed July 31, 2020.

Osilla, K.C., Busum, K.V., Schnyer, C., Larkin, J.W., Eibner, C., \& Mattke, S. (2012). Systematic review of the impact of worksite wellness programs. American Journal of Managed Care, 18(2), e68-81.

Perrin, N.A. (2004). Statistical methods for symptom cluster research: Cluster analysis. Western Institute of Nursing, Portland, OR.

Shay, C.M., Gooding, H.S., Murillo, R., \& Foraker, R. (2015). Understanding and improving cardiovascular health: an update on the American Heart Association's concept of cardiovascular health. Progress in Cardiovascular Diseases, 58(1), 41-49. https://doi.org/10.1016/j.pcad.2015.05.003

Webber, L.S., Rice, J. C., Johnson, C. C., Rose, D., Srinivasan, S. R., \& Berenson, G. S. (2012). Cardiovascular risk factors and physical activity behavior among elementary school personnel: Baseline results from the ACTION! worksite wellness program. The Journal of School Health. 82(9):410-416. https://doi.org/10.1111/j.1746-1561.2012.00716.x

Yang, Q., Cogswell, M.E., Flanders, W.D., Hong, Y., Zhang, Z., Loustalot, F., ... Hu, F.B. (2012). Trends in cardiovascular health metrics and associations with all-cause and CVD mortality among US adults. JAMA, 307, 1273-1283. https://doi.org/10.1001/jama.2012.339

Younus, A., Aneni, E.C., Spatz, E.S., Osondu, C.U., Roberson, L., Ogunmoroti, O., ... Nasir, K. A. (2016). Systematic review of the prevalence and outcomes of ideal cardiovascular health in US and non-US populations. Mayo Clinic Proceedings, (Vol. 91, No. 5, pp. 649-670). Elsevier. https://doi.org/10.1016/j.mayocp.2016.01.019 
Address Author correspondence to:

Heather Carter-Templeton, PhD, RN-BC, FAAN

West Virginia University, School of Nursing

64 Medical Center Drive

Morgantown, WV 26506-9600

heather.cartertempleton@hsc.wvu.edu 